\title{
Encapsulation and Enhanced Delivery of Topoisomerase I Inhibitors in Functionalized Carbon Nanotubes
}

\author{
Sieun Chae, ${ }^{\dagger, \|}$ Dahee Kim, ${ }^{\ddagger}$ Kyung-jin Lee, ${ }^{\ddagger}$ Dasol Lee, ${ }^{\ddagger}$ Young-O Kim, ${ }^{\S}$ Yong Chae Jung,
}

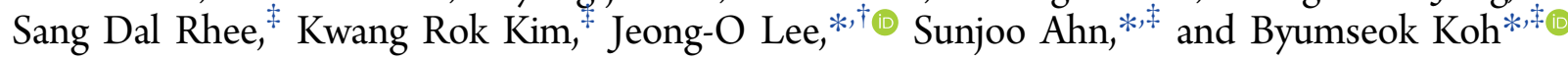

${ }^{\dagger}$ Advanced Materials Division and ${ }^{\ddagger}$ Bio and Drug Discovery Division, Korea Research Institute of Chemical Technology, 141 Gajeong-ro, Yuseong-gu, Daejeon 34114, Republic of Korea

${ }^{\S}$ Institute of Advanced Composite Materials, Korea Institute of Science and Technology, 92 Chudong-ro, Bongdong-eup, Wanju-gun, Jeollabuk-do 55324, Republic of Korea

\section{Supporting Information}

ABSTRACT: The topoisomerase I inhibitors SN-38 and camptothecin (CPT) have shown potent anticancer activity, but water insolubility and metabolic instability limits their clinical application. Utilizing carbon nanotubes as a protective shell for water-insoluble SN-38 and CPT while maintaining compatibility with aqueous media via a carboxylic acidfunctionalized surface can thus be a strategy to overcome this limitation. Through hydrophobic-hydrophobic interactions, SN-38 and CPT were successfully encapsulated in carboxylic acid functionalized single-walled carbon nanotubes and dispersed in water. The resulting cell proliferation inhibition and drug distribution profile inside the cells suggest that these drug-encapsulated carbon nanotubes can serve as a promising delivery strategy for water-insoluble anticancer drugs.

\section{INTRODUCTION}

The FDA-approved topoisomerase I inhibitor irinotecan is used to treat colon and small lung cancer. ${ }^{1-3}$ Its active metabolite SN-38 and analogue camptothecin (CPT) show potent anticancer efficacy and are often considered two of the most effective anticancer agents. ${ }^{4,5}$ Their cytotoxic effect is exerted by blocking the action of topoisomerase I, which is essential for relaxing DNA supercoiling during the cell replication cycle., However, the water insolubility and poor pharmacological profile of $\mathrm{SN}-38$ and $\mathrm{CPT}$ significantly restrict their clinical applications. ${ }^{7,8}$ One approach to overcome this problem is to enhance the drugs' pharmacological profiles by encapsulating them in polymers or nanomaterials functioning as a watercompatible outer shell. ${ }^{9-11}$ Due to their needle-like shape and high aspect ratio, functionalized carbon nanotubes (fCNTs) allow endocytosis or direct penetration across cell membranes. ${ }^{12-16}$ In addition, fCNTs trap hydrophobic molecules in their inner space via hydrophobic-hydrophobic interactions. ${ }^{17-19}$ The carboxylic functional groups on the surface allow their free dispersion in aqueous media without surfactants or dispersants and can serve as a conjugation site for attaching targeting moieties. Moreover, many studies report that compared to nonfunctionalized carbon nanotubes, fCNTs show enhanced clearance ${ }^{20}$ and decreased toxicity, ${ }^{21}$ and can even can be decomposed inside the human body. ${ }^{22,23}$ Biodistribution of fCNTs can also be tracked with endogenous fluorescence emission in the NIR-II window. ${ }^{24,25}$ Therefore, hydrophobic drug molecule encapsulation in fCNTs is a good delivery strategy, as it can protect drugs from external
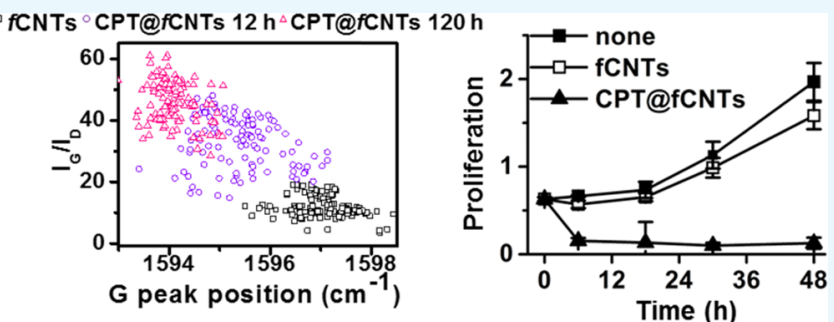

decomposing agents while maintaining compatibility with the aqueous media with traceability. Several careful simulations as well as experimental studies on encapsulating small molecules including drugs in carbon nanotubes have been demonstrated. $^{26-29}$ Although these studies have shown that hydrophobic molecules can be successfully entrapped in the endohedral space of CNTs by simple incubation, ${ }^{17,19}$ studies on the actual delivery of CNT-encapsulated hydrophobic drugs are rare. To enhance SN-38 and CPT delivery and address this unmet research need, we conducted systematic studies on SN-38/CPT encapsulation in fCNTs. In addition, the dispersibility of fCNTs after drug encapsulation in water and the stability in aqueous media and distribution in cells of the encapsulated drugs after delivery were carefully examined to verify the potential of CNT-encapsulated anticancer drugs.

\section{RESULTS}

SN-38 and CPT Encapsulation in fCNTs and Their Dispersion in Aqueous Media. SN-38 and CPT were incubated with $\mathrm{fCNT}$ soot to trap them within the inner cavity of the fCNTs. SN-38 and CPT at $1 \mathrm{mg} / \mathrm{mL}$ in dimethyl sulfoxide (DMSO) were incubated with fCNTs for $120 \mathrm{~h}$. Then, the samples were centrifuged and the supernatant was collected to measure the amount of unencapsulated SN-38 and CPT (F1, Figure 1A,B). Drugs@fCNTs were further washed,

Received: March 5, 2018

Accepted: May 22, 2018

Published: June 1, 2018 
A

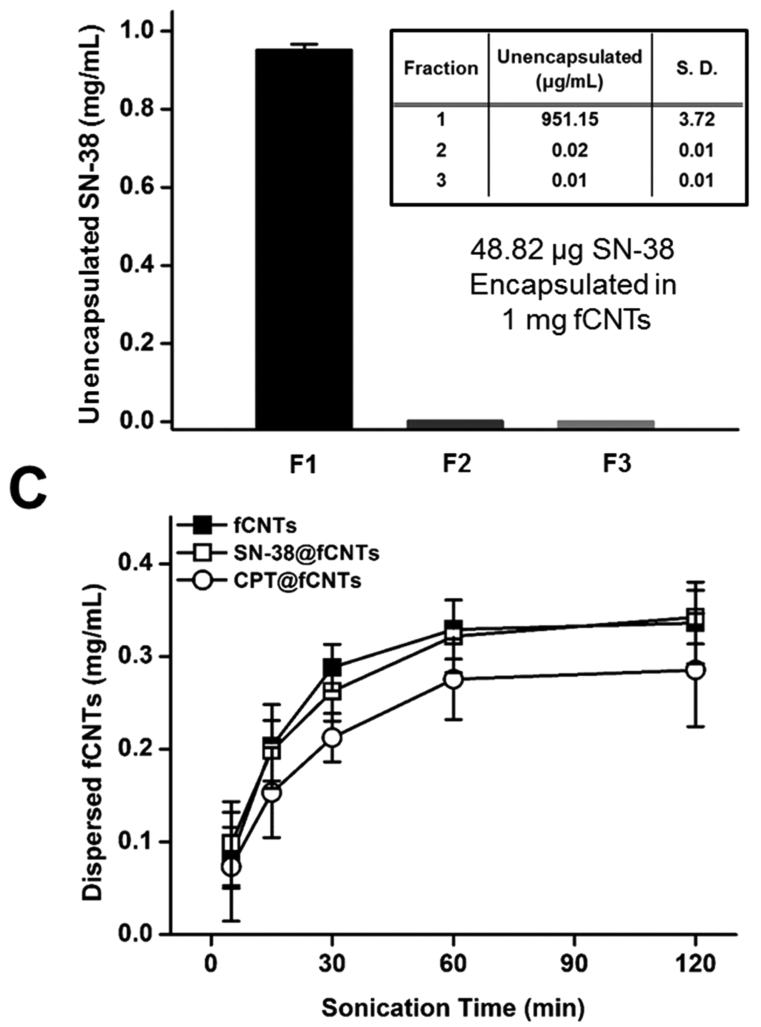

B

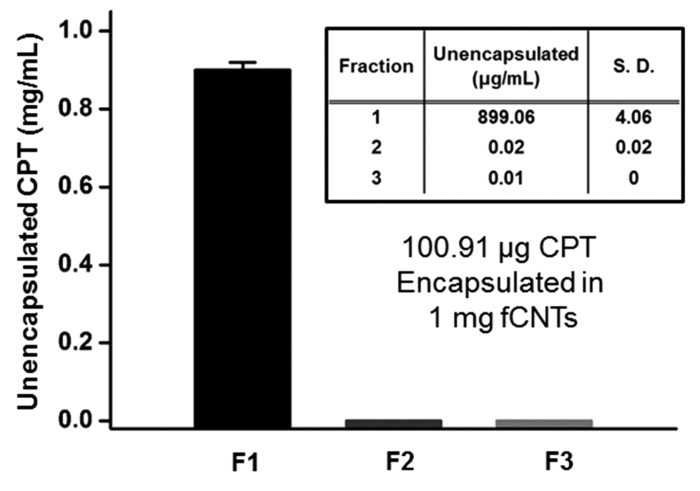

D

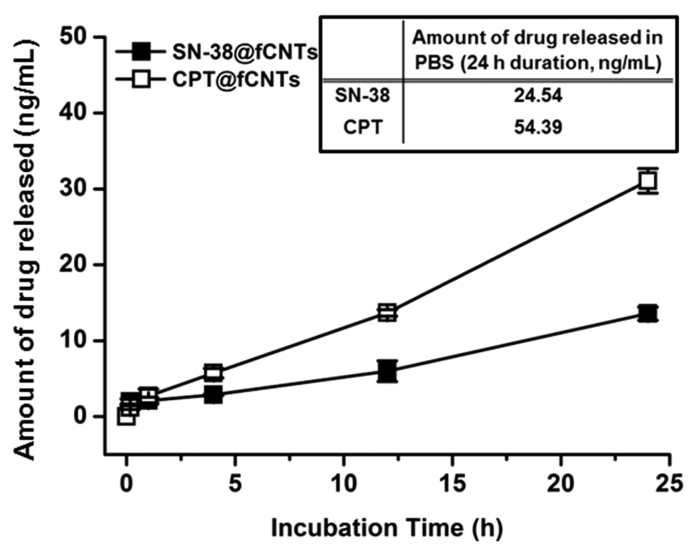

Figure 1. SN-38 and CPT encapsulation in fCNTs. Amount of unencapsulated (A) SN-38 and (B) CPT after incubation with fCNTs. (C) Dispersion of fCNTs in water upon sonication. (D) Amount of SN-38 and CPT released in PBS with stirring. Error bars represent the standard deviation of three replicates.

and the supernatant was collected after each wash (F2 and F3). The data show that $\mathrm{F} 1$ contains the majority of the unencapsulated SN-38 and CPT, followed by F2 and F3. According to an estimation, 48.82 and $100.91 \mu \mathrm{g}$ of $\mathrm{SN}-38$ and CPT were encapsulated on average in $1 \mathrm{mg}$ of fCNTs, respectively (Figure 1A,B). After the encapsulation process, fCNTs containing SN-38 and CPT were dispersed in ultrapure water by bath sonication. The amount of dispersed fCNTs was carefully estimated by subtracting the weight of the undispersed fCNT residue after centrifugation from the starting amount of fCNTs. Similar to the fCNTs without drugs, the amount of SN38- and CPT-encapsulated fCNTs dispersed in water increased with increasing sonication time and reached a maximum of 0.31 mg/mL SN-38@fCNTs and 0.28 mg/mL CPT@fCNTs after 1 $\mathrm{h}$ of sonication (Figure 1C).SN-38@fCNTs and CPT@fCNTs showed less than $10 \%$ deviation in the total amount dispersed compared to the fCNTs without the drugs. To determine the stability of the encapsulated SN-38 and CPT in an aqueous environment, the amount of SN-38 and CPT released from the fCNTs was measured by stirring against Dulbecco's phosphate buffered saline (DPBS) in a dialysis tube. The data suggest that 1.10 and $1.45 \%$ of the total encapsulated SN-38 and CPT, respectively, were released from the fCNTs into DPBS over a $24 \mathrm{~h}$ period (Figure 1D).

Characterization of Drug@fCNTs. To confirm the successful encapsulation of topoisomerase I inhibitors in fCNTs, fCNTs incubated with SN-38 and CPT were characterized with Raman spectroscopy, transmission electron microscopy (TEM), and photoluminescence spectroscopy. Compared to fCNT soot and fCNTs incubated with free
DMSO, SN-38@fCNTs and CPT@fCNTs showed an increased peak intensity of the radial breathing mode (RBM), $\mathrm{G}$ mode, and $\mathrm{G}^{\prime}$ mode with respect to the peak intensity of the D mode (Figure 2A). The peak position shifts of RBM, $\mathrm{G}^{-}$, and $\mathrm{G}^{+}$as well as the two-dimensional (2D) band were also observed after drug encapsulation. Longer incubation $(120 \mathrm{~h})$ of the fCNTs with CPT further increases the peak intensity compared to a shorter incubation time (12 h, Figure $2 \mathrm{~B})$. In addition, the encapsulation of CPT in fCNTs induces a further shift of the G and $2 \mathrm{D}$ peak positions and the $I_{\mathrm{G}} / I_{\mathrm{D}}$ and $I_{2 \mathrm{D}} / I_{\mathrm{D}}$ ratio (Figure $2 \mathrm{C}$ ). Elemental analysis with energy-dispersive spectroscopy (EDS) showed a higher presence of oxygen and nitrogen in SN-38@fCNTs and CPT@fCNTs than in fCNT soot (Figure 2D). Photoluminescence data suggest that the encapsulation of CPT in fCNTs decreased the peak intensity compared to the bare fCNTs (Figure S1).

Drug@fCNTs Delivery to Cancer Cells. To validate fCNTs as a promising drug carrier, we investigated the anticancer efficacy of SN-38 and CPT encapsulated in fCNTs against human cancer cells. SN-38@fCNTs and CPT@fCNTs were effective in inhibiting $\mathrm{PC}-3$ and $\mathrm{LNCaP}$ cell growth compared to equivalent amounts of SN-38 and CPT in water or DMSO (Figure 3A,E). CPT@fCNTs dispersed in water showed comparable inhibition of cancer cell proliferation with CPT solubilized in DMSO (Figure 3B,F). The drug@fCNTsdependent PC-3 and $\mathrm{LNCaP}$ cell growth inhibition data suggest that the anticancer effects of SN-38@fCNTs and CPT@fCNTs are equivalent to those of the same amounts of SN-38 and CPT in DMSO (Figure S2). Western blotting of lysed cells incubated with SN-38@fCNTs and CPT@fCNTs 

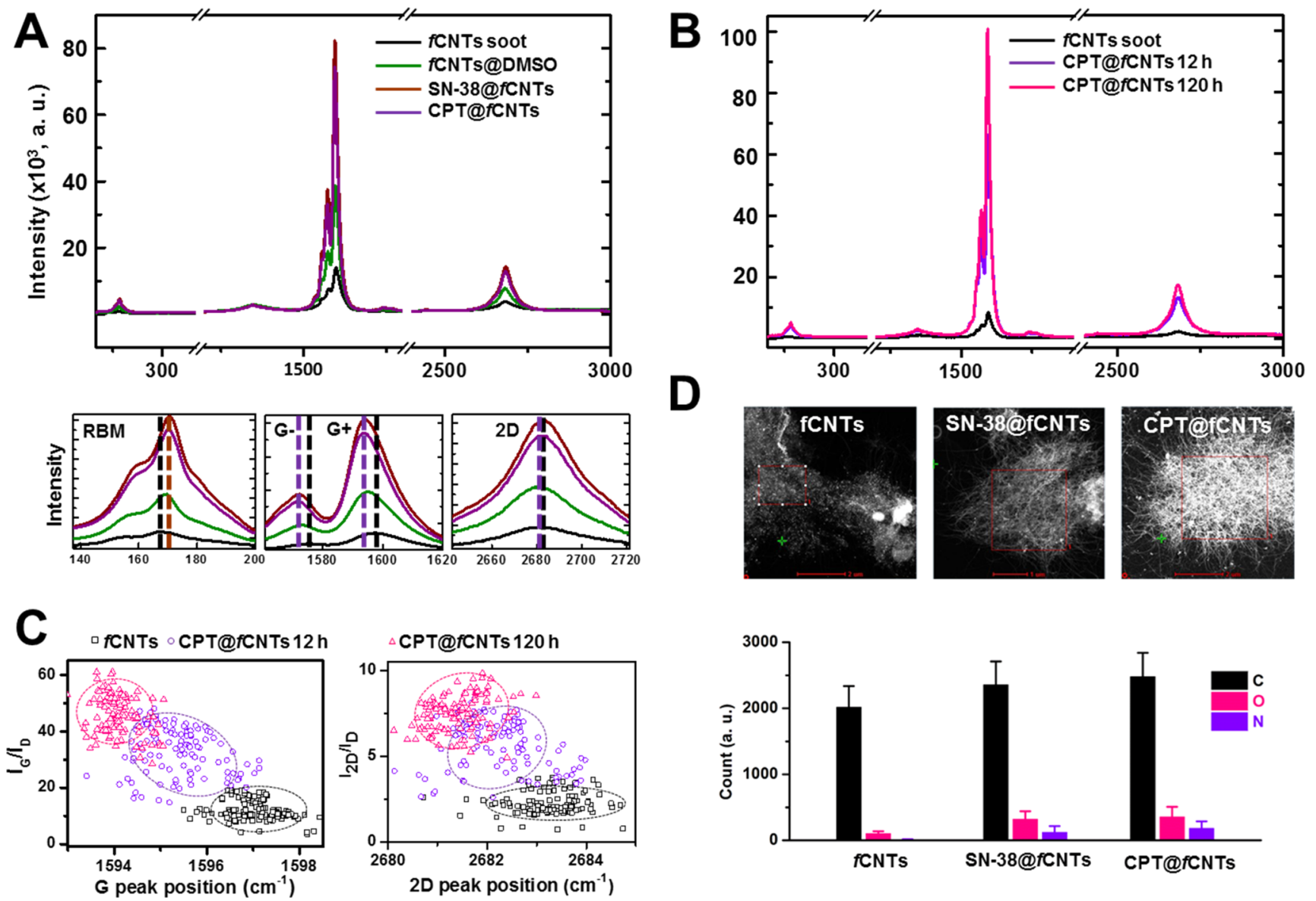

Figure 2. Characterization of drug-encapsulated fCNTs. Raman peak intensity changes after (A) SN-38 and CPT encapsulation and (B) SN-38 encapsulation with varying incubation times. (C) $I_{\mathrm{G}} / I_{\mathrm{D}}$ and $I_{2 \mathrm{D}} / I_{\mathrm{D}}$ ratio changes upon drug encapsulation. (D) TEM images of fCNTs and elemental analysis with energy-dispersive spectroscopy (EDS).

revealed an increase in apoptotic markers (Bax, CC-3, CC-9, and p53), whereas cells incubated with bare fCNTs dispersed in water and $\mathrm{CPT}$ dispersed in water showed minimal change (Figure 3C,G). Cell morphology changes were also observed, as LNCaP and PC-3 cells incubated with CPT@fCNTs showed disintegrated cell membranes and eventual cell death (Figure 3D,H, respectively). The TEM images of PC-3 cells incubated with CPT@fCNTs also showed cell membrane disintegration compared to PC-3 cells without drug treatment (Figure S3).

Drug Distribution in Cancer Cells after Drug@fCNTs Delivery. To determine the destination of fCNT-encapsulated drugs inside cells, the distribution of SN-38 and CPT inside the cells was monitored. Briefly, LNCaP and PC-3 cells incubated with SN-38@fCNTs and CPT@fCNTs were lysed, and the drug distribution in each cellular fraction was analyzed using a cell fractionation kit. The majority of SN-38 after incubation with SN-38@fCNTs was found in the cytoskeletal fraction, followed by the plasma, mitochondria, and ER/Golgi membrane (Figure 4A), whereas CPT was distributed mostly in the membrane, followed by the cytoskeletal and soluble nuclear fractions (Figure 4C). Changes in the distribution profile of $\mathrm{SN}-38$ and $\mathrm{CPT}$ during a $24 \mathrm{~h}$ incubation period showed that the peak concentration of $\mathrm{SN}-38$ in the cytoskeletal fraction at $10 \mathrm{~h}$ gradually decreased, whereas CPT showed a gradual increase in membrane distribution over the $24 \mathrm{~h}$ monitoring period (Figure 4B,D). SN-38 in the other cellular fragments also showed a peak at approximately $10 \mathrm{~h}$ with a stable or gradual decrease in concentration during the next $14 \mathrm{~h}$. In contrast, the CPT distribution in the cytoskeletal and cytoplasmic fractions showed a gradual decrease, whereas the distribution in the nucleic fraction remained relatively constant over the $24 \mathrm{~h}$ monitoring period.

\section{DISCUSSION}

Based on careful studies on the endohedral filling of carbon nanotubes, ${ }^{17-19}$ anticancer drug encapsulation in and delivery from fCNTs were studied. SN-38 and CPT were selected as candidates for fCNT encapsulation, as both of them are potent topoisomerase I inhibitors (the reported $\mathrm{IC}_{50}$ of SN-38 is 120 $\mathrm{nM}$ in an HT29-based cell assay, and the reported $\mathrm{IC}_{50}$ of CPT is $250 \mathrm{nM}$ in an MDA-MB-231-based cell assay; Table S1); ${ }^{4,28}$ however, delivery is an issue, as both drugs are insoluble in water. As the inner cavity of CNTs is hydrophobic, the inner space of CNTs can provide a suitable environment for hydrophobic drugs. The fCNTs were selected as an encapsulating carrier for SN-38 and CPT. They have shown low cellular toxicity with fast clearance and can be dispersed in aqueous media without surfactants or dispersants. ${ }^{30,31}$ The encapsulation of SN-38 and CPT in fCNTs was achieved by a simple incubation method reported by another group; ${ }^{17-19}$ however, very gentle stirring was applied, as intensive stirring induces the dispersion of fCNTs in DMSO. A $120 \mathrm{~h}$ incubation in the presence of SN-38 and CPT resulted in topoisomerase I inhibitor-encapsulated fCNTs, as confirmed by various 


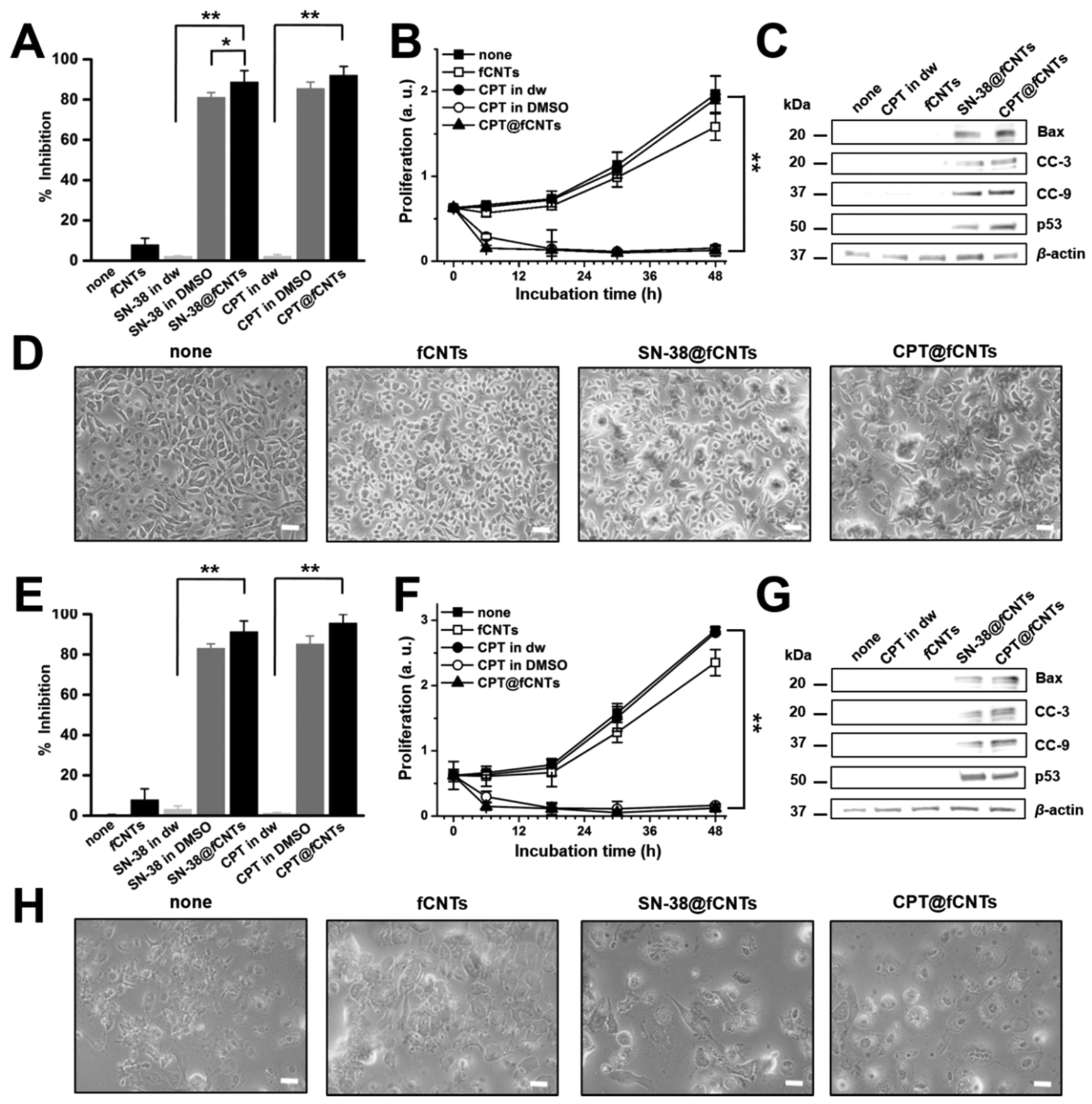

Figure 3. Anticancer effect of drug@fCNTs. Inhibition of (A) LNCaP and (E) PC-3 cell growth. Proliferation inhibition of (B) LNCaP and (F) PC3 cells and apoptotic protein expression of (C) LNCaP and (G) PC-3 cells after incubation with drug@fCNTs. Microscopic images of (D) LNCaP and (H) PC-3 after drug@fCNTs incubation. Statistical analysis was performed using an unpaired $t$ test, where $*_{p}<0.05$, $* * p<0.01$. Scale bars represent $10 \mu \mathrm{m}$.

techniques (Figure 2). Although the $\log P$ of $\mathrm{SN}-38$ is similar or slightly higher than that of CPT, the amount of CPT encapsulated in fCNTs $(100.91 \mu \mathrm{g})$ is higher than that of SN-38 $(48.82 \mu \mathrm{g})$ under identical conditions. Campo et al. conduct careful studies on CNT endohedral filling with more than 20 different molecules. ${ }^{17} \mathrm{Cis}$ and cis/trans mixture of decalin showed different absorbance for reasons include in packing efficiencies. ${ }^{17}$ This suggests that small changes in molecular structure can influence the CNT endohedral filling efficiencies. Difference in encapsulation efficiency is likely due to extra ethyl and hydroxyl group on $\mathrm{SN}-38$ despite only $\sim 12.6 \%$ (392.4 vs 348.4, Table S1) difference in the molecular weight. Decrease in photoluminescence intensity after encapsulation was observed, and we speculate that irregularity in encapsulation may reduce fCNT properties (Figure S1). After encapsulation, the degree of SN-38@fCNTs and CPT@ fCNTs dispersion in aqueous media was examined. Compared to bare fCNTs, SN-38-encapsulated fCNTs and CPTencapsulated fCNTs showed only a minor decrease in dispersion (1 and 9\% decrease, respectively), suggesting that neither SN-38 nor CPT encapsulation induces significant modification of the carboxylic functional groups on the surface of the fCNTs. Next, the stability of the fCNT-encapsulated drugs in aqueous media was examined. To serve as a drug 

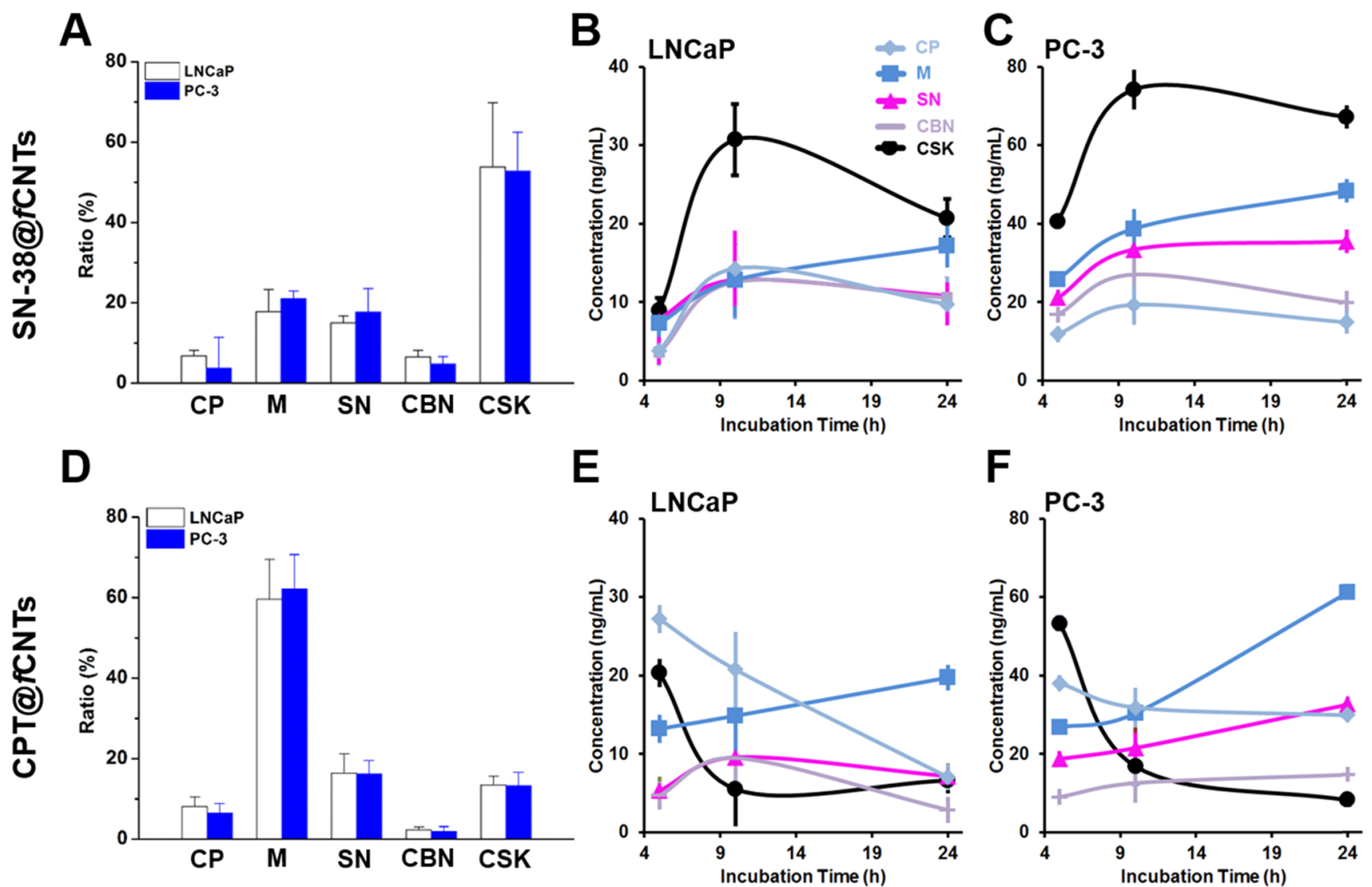

Figure 4. Drugs released from fCNTs inside cancer cells. (A) \% SN-38 and (D) CPT detected in each cell fraction. Concentration of SN-38 detected after a designated incubation time from each cellular fraction of (B) LNCaP and (C) PC-3 cells. Concentration of CPT detected after a designated period of time from each cellular fraction of (E) LNCaP and (F) PC-3 cells. CP, cytoplasmic extract; M, plasma, mitochondria, and ER/Golgi membrane extract; $\mathrm{SN}$, soluble nuclear extract; CBN, chromatic bound nuclear extract; CSK, cytoskeletal extract.

carrier, the fCNTs should retain the encapsulated drug in an aqueous environment until they reach their targets. By monitoring the amount of drug released during a $24 \mathrm{~h}$ incubation period, we observed that only a fraction of the encapsulated SN-38 and CPT was released from the fCNTs ( 1.01 and $1.28 \%$, respectively); therefore, fCNTs can serve as a water-compatible carrier for hydrophobic drugs. After the encapsulation process, the drug-encapsulating fCNTs were characterized by Raman spectroscopy and TEM. Figure $2 \mathrm{~A}$ shows that the peak intensity of the RBM, G, and 2D peaks significantly increased after the fCNTs were incubated with free DMSO and further increased after they were incubated with $\mathrm{SN}-38$ and CPT, whereas the intensity of the $\mathrm{D}$ mode remained almost the same. We assumed that the enhanced $I_{\mathrm{RBM}} / I_{\mathrm{D}}, I_{\mathrm{G}} / I_{\mathrm{D}}$, and $I_{2 \mathrm{D}} / I_{\mathrm{D}}$ ratios after the encapsulation process were due to the destroyed amorphous carbon included in the fCNTs, thereby diminishing the shielding effect in the Raman spectra. ${ }^{32}$ In addition to the peak intensity change, the peak positions of the RBM, G $\mathrm{G}^{-}$, and $\mathrm{G}^{+}$bands shifted after the encapsulation process. Specifically, the peak shift of the G mode was from $\sim 1598.99$ to $1595.50 \mathrm{~cm}^{-1}$ and that of the RBM mode was from 167.32 to $170.55 \mathrm{~cm}^{-1}$ with drug encapsulation. Such effects may be attributed to the charge fluctuations and structural lattice deformations of the fCNTs caused by external molecules. ${ }^{33,34}$ Also, the peak shifts of RBM and G bands were observed with DMSO treatment; and this can be due to doping effect from amorphous carbon residues were removed by DMSO or changing in the electronic states of fCNTs. We also cannot completely rule out the effects of residual DMSO encapsulated in fCNTs. We further acquired the Raman spectra of CPT@fCNT samples with increasing encapsulation time (Figure $2 \mathrm{~B}$ ) and plotted the $I_{\mathrm{G}} / I_{\mathrm{D}}$ and $\mathrm{G}$ peak position and $I_{2 \mathrm{D}} /$ $I_{\mathrm{D}}$ and $2 \mathrm{D}$ peak position against each other with 121 spectra for each sample (Figure $2 \mathrm{C}$ ). As the incubation time increased, a gradual increase in the $I_{\mathrm{G}} / I_{\mathrm{D}}$ and $I_{2 \mathrm{D}} / I_{\mathrm{D}}$ ratios and a gradual decrease in the $\mathrm{G}$ and $2 \mathrm{D}$ peak positions were found, indicating a possible encapsulation of the drug molecules in fCNTs with increase in the incubation time. The elemental analysis with EDS also suggests the successful encapsulation of SN-38 and $\mathrm{CPT}$ in the fCNTs. Compared to that of the bare fCNTs, the surfaces of the SN-38- and CPT-encapsulated fCNTs showed significant concentrations of carbon $(\mathrm{C})$, nitrogen $(\mathrm{N})$, and oxygen $(\mathrm{O})$ atoms. As the amount of CPT encapsulated inside the fCNTs is higher than the amount of SN-38 encapsulated, more $\mathrm{N}$ and $\mathrm{O}$ atoms were detected on CPT@fCNTs than on SN-38@fCNTs despite SN-38 featuring an additional O atom (SN-38 and CPT carry the same number of $\mathrm{N}$ atoms). After confirming the successful encapsulation and dispersion of drug@fCNTs in aqueous media, we examined the anticancer activity of drug@fCNTs. The LNCaP and PC-3 cell lines were selected as therapeutic target cells, as topoisomerase I inhibitors showed promising anticancer efficacy against colon and small lung cancer, yet their potency against prostate cancer is not confirmed. ${ }^{35}$ Compared to equivalent amounts of SN-38/CPT nonhomogeneously dispersed in water, SN-38@fCNTs and CPT@fCNTs showed $>90 \%$ more inhibition of cancer cell 
proliferation. Bare fCNTs dispersed in water induce 7.89 and 7.93\% decrease in LNCaP and PC-3 cell viability, respectively, suggesting that the anticancer efficacy of drug@fCNTs is mostly from the encapsulated drugs released and not from the fCNTs.SN-38@fCNTs and CPT@fCNTs showed similar cancer cell proliferation inhibition profiles with $\mathrm{SN}-38 / \mathrm{CPT}$ solubilized in DMSO; however, they have an advantage over DMSO-solubilized drugs: drug-carrying fCNTs have better compatibility with aqueous media. Apoptosis induced by drug@fCNTs was confirmed by Western blotting of lysed cancer cells incubated with SN-38@fCNTs and CPT@fCNTs. Increased expression levels of the apoptotic markers Bax, CC-3, CC-9, and p53 were observed in PC-3 and LNCaP cells incubated with SN-38@fCNTs and CPT@fCNTs, whereas cells incubated with bare fCNTs or CPT dispersed in water showed minimal expression levels. Two main CNT cell entry mechanisms were proposed by previous studies: endocytosis and direct penetration. ${ }^{36,37}$ Although some drug@fCNT may enter cells via direct penetration, endocytosis blocking experiment at $4{ }^{\circ} \mathrm{C}$ suggests that endocytosis is drug@ fCNT's main cellular entry mechanism (Figure S4). After confirming the efficacy of drug@fCNTs, the distribution of SN38 and CPT after drug@fCNTs cell entry was examined. Interestingly, SN-38 and CPT showed markedly different distributions, as the concentration of SN-38 in the cytoskeletal fraction of cells increased over $10 \mathrm{~h}$ and remained high, whereas CPT showed a gradual increase in concentration in the plasma, mitochondria, and endoplasmic reticulum/Golgi membranes. SN-38 and CPT showed similar distribution profiles in PC-3 and LNCaP cells, suggesting that the distribution in each cellular fraction is more dependent on the type of drug. The structural difference between SN-38 and CPT may play a major role, but the detailed mechanism underlying this difference in the distribution remains unclear and is a subject for further research. In conclusion, the encapsulation of the hydrophobic topoisomerase I inhibitors SN-38 and CPT in the fCNTs is successfully demonstrated. With 4.88 (SN-38) and 10.09 (CPT) \% encapsulation efficiencies, drug@fCNTs can be successfully dispersed without losing a significant amount of the drugs in aqueous media. Our result suggests that $\mathrm{SN}-38$ and CPT encapsulated in fCNTs can be successfully delivered to and released in cancer cells and can exert their anticancer effect while maintaining their water compatibility inside the fCNT shell during delivery.

\section{EXPERIMENTAL SECTION}

Materials. fCNTs (>90\% carbon basis), SN-38 (>98\% purity), and CPT (>90\% purity) were purchased from SigmaAldrich (St. Louis, MO). PC-3 and LNCaP cell lines were purchased from American Type Culture Collection (Manassas, VA). Ultrapure water (molecular biology grade, $18 \mathrm{M} \Omega$ resistivity), RPMI-1640 medium, fetal bovine serum (FBS), $10 \times$ Tris-buffered saline, and Dulbecco's phosphate buffered saline (DPBS) were purchased from Welgene (Gyeongsan, Korea). TrypLE express, penicillin-streptomycin, and a subcellular protein fractionation kit were purchased from Thermo Fisher Scientific, Inc. (Waltham, MA). Cell culture flasks $\left(25\right.$ and $\left.75 \mathrm{~cm}^{2}\right)$ and cell culture dishes $(60 \times 15 \mathrm{~mm})$ were purchased from Corning (Corning, NY). A cell counting kit-8 (CCK-8) was purchased from Dojindo Laboratories (Kumamoto, Japan). Precast gel for Western blotting was purchased from Bio-Rad Laboratories, Inc. (Hercules, CA). Western blot detection reagent was purchased from GE
Healthcare (Chicago, IL). Bax, cleaved caspase 3 (CC-3), cleaved caspase 8 (CC-8), and $\beta$-actin antibodies were purchased from Cell Signaling Technology (Danvers, MA). All other reagents were purchased from Sigma-Aldrich.

Topoisomerase I Inhibitor Encapsulation in fCNTs. Encapsulation of topoisomerase I inhibitor was achieved by simple incubation with mild agitation described previously. ${ }^{17-19}$ More specifically, $1 \mathrm{mg}$ of SN-38 and CPT was solubilized in 1 $\mathrm{mL}$ of dimethyl sulfoxide (DMSO, ACS grade > 99.9\%) and incubated with $1 \mathrm{mg}$ of fCNTs in capped flat-bottom test tubes with a magnetic stir bar. The samples were gently stirred in the dark for 5 days at room temperature unless described otherwise. After stirring, the samples were centrifuged at 15 $000 \mathrm{rpm}$ for $1 \mathrm{~h}$, and the unencapsulated (free) SN-38/CPTcontaining supernatant was collected. The residue was intensively washed, followed by centrifugation at $15000 \mathrm{rpm}$ for $1 \mathrm{~h}$. The supernatant was collected for analysis, and the washing step was repeated twice. After the final washing step, the samples were dried to yield SN-38@fCNTs and CPT@ fCNTs.

Characterization of Topoisomerase I Inhibitor-Encapsulated fCNTs. Raman spectroscopy was conducted using an Invia Raman spectrometer equipped with a $514 \mathrm{~nm}$ laser (Renishaw, Gloucestershire, U.K.). Transmission electron microscopy (TEM) images of fCNTs and PC-3 cell images after CPT@fCNTs delivery were obtained by an FEI Themis TEM (Hillsboro, OR). Near-infrared (NIR) fluorescence spectra were obtained through a HORIBA Jobin Yvon Nanolog NIR spectrophotometer (Kyoto, Japan) equipped with a $450 \mathrm{~W}$ xenon arc lamp and a liquid nitrogen-cooled InGaAs detector. The excitation wavelengths ranged from 400 to $800 \mathrm{~nm}$ with 2 $\mathrm{nm}$ increments, and the emission wavelengths ranged from 850 to $1600 \mathrm{~nm}$ with $1.5 \mathrm{~nm}$ increments. The fluorescence intensities were recorded with $20 \mathrm{~s}$ of integration per scan and slit widths for the excitation and emission of $14 \mathrm{~nm}$ and 40 $\mathrm{nm}$, respectively.

Dispersion of Topoisomerase I Inhibitor@fCNTs and Release of SN-38/CPT in Aqueous Media. One milliliter of molecular biology grade ultrapure water was added to $1 \mathrm{mg}$ of SN-38/CPT-encapsulated fCNTs and sonicated for $1 \mathrm{~h}$ in water bath $(50 \mathrm{~W})$ with ice to prevent overheating. After sonication, the samples were centrifuged at $15000 \mathrm{rpm}$ for $1 \mathrm{~h}$. Dispersed drug@fCNTs were collected, and undispersed CNT residues were carefully collected for weight measurement. To test the release of the encapsulated SN-38/CPT in aqueous media, $3 \mathrm{~mL}$ of dispersed drug@fCNTs in water was loaded in a dialysis tube and stirred in the presence of $20 \mathrm{~mL}$ of DPBS. After $0.5,1,4,12$, and $24 \mathrm{~h}$, DBPS was collected, and the concentrations of SN-38 and CPT released in DPBS were analyzed with an liquid chromatography-mass spectrometry (LC-MS/MS) consisting of an Agilent 1200 series HPLC system (Agilent Technologies, Santa Clara, CA) equipped with an API 4000 QTRAP hybrid triple quadrupole/linear ion trap mass spectrometer (AB Sciex Pte Ltd., Foster City, CA).

Cell Culture and Topoisomerase I Inhibitor Delivery. The prostate cancer cell line PC-3 and the LNCaP cell line were cultured in RPMI-1640 medium supplemented with $10 \%$ FBS and $1 \%$ penicillin-streptomycin at $37{ }^{\circ} \mathrm{C}$ in a $5 \% \mathrm{CO}_{2}$ incubator. For a stable delivery, $2 \times 10^{5} \mathrm{PC}-3 / \mathrm{LNCaP}$ cells were cultured on a 24-well plate and preincubated for $24 \mathrm{~h}$. Then, $100 \mu \mathrm{L}$ of SN-38@fCNTs $(\sim 1.5 \mu \mathrm{g}$ of SN-38) and CPT@fCNTs $(\sim 2.5 \mu \mathrm{g}$ of CPT) were mixed with $400 \mu \mathrm{L}$ of RPMI-1640 medium and incubated with cells for designated 
times. Equivalent amounts of SN-38 and CPT solubilized in DMSO/dispersed in water were mixed with RPMI-1640 and added to the cells. The cells were carefully washed with DPBS, and the cell proliferation was analyzed with a CCK-8 and a microplate reader (Molecular Devices SpectraMax i3, Sunnyvale, CA) according to the manufacturer's instructions. Bax, $\mathrm{CC}-3$, and $\mathrm{CC}-8$ antibodies were used to monitor the apoptotic effect of SN-38 and CPT with Western blotting. For the drug@ fCNTs dose-dependent cell proliferation inhibition assay, 10, 20, 50, 100, and $200 \mu \mathrm{L}$ of SN-38@fCNTs/CPT@fCNTs and an equivalent amount of SN-38/CPT in water or DMSO were mixed with RPMI-1640 to a final volume of $500 \mu \mathrm{L}$ and incubated with $\mathrm{LNCaP} / \mathrm{PC}-3$ cells as described above. The cell images were obtained using an Olympus IX 73 inverted microscope (Tokyo, Japan) and an FEI Themis TEM.

Distribution of SN-38 and CPT in Cells after Delivery. PC-3 and LNCaP cells at a density of $2 \times 10^{6}$ were preincubated in $60 \times 15 \mathrm{~mm}$ cell culture dishes for $48 \mathrm{~h}$. After a careful wash with DPBS, the cells were incubated with 1 mL of SN-38@fCNTs and CPT@fCNTs mixed with $4 \mathrm{~mL}$ of RPMI-1640 medium. After 5, 10, and $24 \mathrm{~h}$ of incubation, the medium was removed and carefully washed twice with DPBS. SN-38 and CPT subcellular fractions were obtained using a subcellular protein fractionation kit (Thermo Fisher Scientific). The concentrations of SN-38 and CPT in the fractions were measured by an LC-MS/MS system.

\section{ASSOCIATED CONTENT}

\section{S Supporting Information}

The Supporting Information is available free of charge on the ACS Publications website at DOI: 10.1021/acsomega.8b00399.

Fluorescence-excitation color maps, cell \% inhibition, TEM image of cells after incubation, endocytosis blocking experimental data (PDF)

\section{AUTHOR INFORMATION}

\section{Corresponding Authors}

*E-mail: jolee@krict.re.kr. Phone: +82-42-860-7336. Fax: +8242-860-7508 (J.-O.L.).

*E-mail: sahn@krict.re.kr. Phone: +82-42-860-7170. Fax: +8242-860-7459 (S.A.).

*E-mail: bkoh@krict.re.kr. Phone: +82-42-860-7465. Fax: +8242-861-4146 (B.K.).

\section{ORCID}

Jeong-O Lee: 0000-0002-7343-4892

Byumseok Koh: 0000-0002-1106-1891

\section{Present Address}

"Department of Materials Science and Engineering, University of Michigan, Ann Arbor, Michigan 48109, United States (S.C.).

\section{Author Contributions}

The manuscript was written through contributions of all the authors. All the authors have given approval to the final version of the manuscript.

\section{Notes}

The authors declare no competing financial interest.

\section{ACKNOWLEDGMENTS}

This research was supported by the Korea Research Institute of Chemical Technology core project (KK1703-F00).

\section{REFERENCES}

(1) Rougier, P.; Bugat, R.; Douillard, J. Y.; Culine, S.; Suc, E.; Brunet, P.; Becouarn, Y.; Ychou, M.; Marty, M.; Extra, J. M.; Bonneterre, J.; Adenis, A.; Seitz, J. F.; Ganem, G.; Namer, M.; Conroy, T.; Negrier, S.; Merrouche, Y.; Burki, F.; Mousseau, M.; Herait, P.; Mahjoubi, M. Phase II Study of Irinotecan in the Treatment of Advanced Colorectal Cancer in Chemotherapy-Naive Patients and Patients Pretreated with Fluorouracil-Based Chemotherapy. J. Clin. Oncol. 1997, 15, 251-260.

(2) Hanna, N.; Bunn, P. A.; Langer, C.; Einhorn, L.; Guthrie, T.; Beck, T.; Ansari, R.; Ellis, P.; Byrne, M.; Morrison, M.; Hariharan, S.; Wang, B.; Sandler, A. Randomized Phase III Trial Comparing Irinotecan/Cisplatin With Etoposide/Cisplatin in Patients With Previously Untreated Extensive-Stage Disease Small-Cell Lung Cancer. J. Clin. Oncol. 2006, 24, 2038-2043.

(3) Tung, W. L.; Wang, Y.; Gout, P. W.; Liu, D. M.; Gleave, M.; Wang, Y. Use of irinotecan for treatment of small cell carcinoma of prostate. Prostate 2011, 71, 675-681.

(4) Kawato, Y.; Aonuma, M.; Hirota, Y.; Kuga, H.; Sato, K. Intracellular Roles of SN-38, a Metabolite of the Camptothecin Derivative CPT-11, in the Antitumor Effect of CPT-11. Cancer Res. 1991, 51, 4187-4191.

(5) Hsiang, Y. H.; Hertzberg, R.; Hecht, S.; Liu, L. F. Camptothecin. J. Biol. Chem. 1985, 260, 14873-14878.

(6) Hsiang, Y.-H.; Lihou, M. G.; Liu, L. F. Arrest of Replication Forks by Drug-Stabilized Topoisomerase I-DNA Cleavable Complexes as a Mechanism of Cell Killing by Camptothecin. Cancer Res. 1989, 49, 5077-5082.

(7) Kunimoto, T.; Nitta, K.; Tanaka, T.; Uehara, N.; Baba, H.; Takeuchi, M.; Yokokura, T.; Sawada, S.; Miyasaka, T.; Mutai, M. Antitumor Activity of 7-Ethyl-10-[4-(1-Piperidino)-1-Piperidino]Carbonyloxycamptothecin, a Novel Water-Soluble Derivative of Camptothecin, against Murine Tumors. Cancer Res. 1987, 47, 59445947.

(8) Stella, V. J.; Nti-Addae, K. W. Prodrug Strategies to Overcome Poor Water Solubility. Adv. Drug Delivery Rev. 2007, 59, 677-694.

(9) Liu, Z.; Robinson, J. T.; Sun, X.; Dai, H. PEGylated Nanographene Oxide for Delivery of Water-Insoluble Cancer Drugs. J. Am. Chem. Soc. 2008, 130, 10876-10877.

(10) Zhang, J. A.; Xuan, T.; Parmar, M.; Ma, L.; Ugwu, S.; Ali, S.; Ahmad, I. Development and Characterization of a Novel LiposomeBased Formulation of SN-38. Int. J. Pharm. 2004, 270, 93-107.

(11) Kolhatkar, R. B.; Swaan, P.; Ghandehari, H. Potential Oral Delivery of 7-Ethyl-10-Hydroxy-Camptothecin (SN-38) Using Poly(Amidoamine) Dendrimers. Pharm. Res. 2008, 25, 1723-1729.

(12) Guo, C.; Al-Jamal, W. T.; Toma, F. M.; Bianco, A.; Prato, M.; Al-Jamal, K. T.; Kostarelos, K. Design of Cationic Multiwalled Carbon Nanotubes as Efficient SiRNA Vectors for Lung Cancer Xenograft Eradication. Bioconjugate Chem. 2015, 26, 1370-1379.

(13) Wang, Y.; Bahng, J. H.; Che, Q.; Han, J.; Kotov, N. A. Anomalously Fast Diffusion of Targeted Carbon Nanotubes in Cellular Spheroids. ACS Nano 2015, 9, 8231-8238.

(14) Devarajan, P. V.; Jain, S. Targeted Drug Delivery: Concepts and Design, 1st ed.; Devarajan, P. V., Jain, S., Eds.; Springer: New York, 2015; pp 615-649.

(15) Klumpp, C.; Kostarelos, K.; Prato, M.; Bianco, A. Functionalized Carbon Nanotubes as Emerging Nanovectors for the Delivery of Therapeutics. Biochim. Biophys. Acta, Biomembr. 2006, 1758, 404-412.

(16) Hong, G.; Diao, S.; Antaris, A. L.; Dai, H. Carbon Nanomaterials for Biological Imaging and Nanomedicinal Therapy. Chem. Rev. 2015, 115, 10816-10906.

(17) Campo, J.; Piao, Y.; Lam, S.; Stafford, C. M.; Streit, J. K.; Simpson, J. R.; Walker, A. R. H.; Fagan, J. A. Enhancing Single-Wall Carbon Nanotube Properties through Controlled Endohedral Filling. Nanoscale Horiz. 2016, 1, 317-324.

(18) Mehrjouei, E.; Akbarzadeh, H.; Shamkhali, A. N.; Abbaspour, M.; Salemi, S.; Abdi, P. Delivery of Cisplatin Anti-Cancer Drug from Carbon, Boron Nitride, and Silicon Carbide Nanotubes Forced by AgNanowire: A Comprehensive Molecular Dynamics Study. Mol. Pharmaceutics 2017, 14, 2273-2284. 
(19) Li, J.; Qi Yap, S.; Fei Chin, C.; Tian, Q.; Lee Yoong, S.; Pastorin, G.; Han Ang, W. Platinum(Iv) Prodrugs Entrapped within Multiwalled Carbon Nanotubes: Selective Release by Chemical Reduction and Hydrophobicity Reversal. Chem. Sci. 2012, 3, 2083-2087.

(20) Liu, Z.; Davis, C.; Cai, W.; He, L.; Chen, X.; Dai, H. Circulation and Long-Term Fate of Functionalized, Biocompatible Single-Walled Carbon Nanotubes in Mice Probed by Raman Spectroscopy. Proc. Natl. Acad. Sci. U.S.A. 2008, 105, 1410-1415.

(21) Vardharajula, S.; Ali, S. Z.; Tiwari, P. M.; Eroğlu, E.; Vig, K.; Dennis, V. A.; Singh, S. R. Functionalized carbon nanotubes: biomedical applications. Int. J. Nanomed. 2012, 7, 5361-5374.

(22) Allen, B. L.; Kichambare, P. D.; Gou, P.; Vlasova, I. I.; Kapralov, A. A.; Konduru, N.; Kagan, V. E.; Star, A. Biodegradation of SingleWalled Carbon Nanotubes through Enzymatic Catalysis. Nano Lett. 2008, 8, 3899-3903.

(23) Allen, B. L.; Kotchey, G. P.; Chen, Y.; Yanamala, N. V. K.; KleinSeetharaman, J.; Kagan, V. E.; Star, A. Mechanistic Investigations of Horseradish Peroxidase-Catalyzed Degradation of Single-Walled Carbon Nanotubes. J. Am. Chem. Soc. 2009, 131, 17194-17205.

(24) Welsher, K.; Liu, Z.; Sherlock, S. P.; Robinson, J. T.; Chen, Z.; Daranciang, D.; Dai, H. A Route to Brightly Fluorescent Carbon Nanotubes for Near-Infrared Imaging in Mice. Nat. Nanotechnol. 2009, $4,773-780$

(25) Hong, G.; Antaris, A. L.; Dai, H. Near-Infrared Fluorophores for Biomedical Imaging. Nat. Biomed. Eng. 2017, 1, No. 0010.

(26) Su, Z.; Zhu, S.; Donkor, A. D.; Tzoganakis, C.; Honek, J. F. Controllable Delivery of Small-Molecule Compounds to Targeted Cells Utilizing Carbon Nanotubes. J. Am. Chem. Soc. 2011, 133, 68746877.

(27) Xue, Q.; Jing, N.; Chu, L.; Ling, C.; Zhang, H. Release of Encapsulated Molecules from Carbon Nanotubes Using a Displacing Method: A MD Simulation Study. RSC Adv. 2012, 2, 6913-6920.

(28) Chaban, V. V.; Prezhdo, O. V. Water Boiling Inside Carbon Nanotubes: Toward Efficient Drug Release. ACS Nano 2011, 5, 56475655.

(29) Chaban, V. V.; Savchenko, T. I.; Kovalenko, S. M.; Prezhdo, O. V. Heat-Driven Release of a Drug Molecule from Carbon Nanotubes: A Molecular Dynamics Study. J. Phys. Chem. B 2010, 114, 1348113486.

(30) Jones, C. B.; Clements, M. K.; Wasi, S.; Daoud, S. S. Sensitivity to Camptothecin of Human Breast Carcinoma and Normal Endothelial Cells. Cancer Chemother. Pharmacol. 1997, 40, 475-483.

(31) Heister, E.; Lamprecht, C.; Neves, V.; Tîlmaciu, C.; Datas, L.; Flahaut, E.; Soula, B.; Hinterdorfer, P.; Coley, H. M.; Silva, S. R. P.; McFadden, J. Higher Dispersion Efficacy of Functionalized Carbon Nanotubes in Chemical and Biological Environments. ACS Nano 2010, 4, 2615-2626.

(32) Hu, Z.; Zhao, J.; Song, Z.; Yang, C. Filled and Peptide-Modified Single-Walled Carbon Nanotubes: Synthesis, Characterization, and in Vitro Test for Cancer Cell Targeting. RSC Adv. 2015, 5, 1679216800.

(33) Dresselhaus, M. S.; Dresselhaus, G.; Saito, R.; Jorio, A. Raman Spectroscopy of Carbon Nanotubes. Phys. Rep. 2005, 409, 47-99.

(34) Fatini, C.; Jorio, A.; Souza, M.; Strano, M. S.; Dresselhaus, M. S.; Pimenta, M. A. Optical Transition Energies for Carbon Nanotubes from Resonant Raman Spectroscopy: Environment and Temperature Effects. Phys. Rev. Lett. 2004, 93, No. 147406.

(35) Reese, D. M.; Tchekmedyian, S.; Chapman, Y.; Prager, D.; Rosen, P. J. A Phase II Trial of Irinotecan in Hormone-Refractory Prostate Cancer. Invest. New Drugs 1998, 16, 353-359.

(36) Kam, N. W. S.; Dai, H. Carbon Nanotubes as Intracellular Protein Transporters: Generality and Biological Functionality. J. Am. Chem. Soc. 2005, 127, 6021-6026.

(37) Kraszewski, S.; Picaud, F.; Elhechmi, I.; Gharbi, T.; Ramseyer, C. How Long a Functionalized Carbon Nanotube Can Passively Penetrate a Lipid Membrane. Carbon 2012, 50, 5301-5308. 